\title{
Localized rainbows in the QCD phase diagram
}

\author{
J. Maelger, ${ }^{1,2}$ U. Reinosa, ${ }^{1}$ and J. Serreau ${ }^{2}$ \\ ${ }^{1}$ Centre de Physique Théorique (CPHT), CNRS, Ecole Polytechnique, Institut Polytechnique de Paris, \\ Route de Saclay, F-91128 Palaiseau, France \\ ${ }^{2}$ Astro-Particule et Cosmologie (APC), CNRS UMR 7164, Université Paris Diderot, 10, \\ rue Alice Domon et Léonie Duquet, 75205 Paris Cedex 13, France
}

(Received 11 March 2019; revised manuscript received 5 September 2019; published 29 January 2020)

\begin{abstract}
We study the phase diagram of strongly interacting matter with light quarks using a recently proposed, small parameter approach to infrared QCD in the Landau gauge. This is based on an expansion with respect to both the inverse number of colors and the pure Yang-Mills coupling in the presence of a Curci-Ferrari mass term. At leading order, this leads to the well-known rainbow equation for the quark propagator with a massive gluon propagator and a tree-level quark-gluon vertex. We solve the latter at nonzero temperature and chemical potential using a simple semianalytic approximation known to capture the essence of chiral symmetry breaking in the vacuum. In the chiral limit, we find a tricritical point which becomes a critical end point in the presence of a nonzero bare quark mass, in agreement with the results of nonperturbative functional methods and model calculations.
\end{abstract}

DOI: 10.1103/PhysRevD.101.014028

\section{INTRODUCTION}

Hadronic matter is expected to present a rich phase structure when submitted to sufficiently high energy and baryonic densities, large magnetic fields, etc., as encountered in various environments such as the early Universe, ultradense astrophysical objects, or relativistic heavy ion collisions in the laboratory [1-3]. Unraveling the phase diagram of quantum chromodynamics (QCD) at nonzero temperature $T$ and baryonic chemical potential $\mu_{B}$ is a major challenge both experimentally and theoretically. At vanishing chemical potential, first principle lattice simulations unambiguously demonstrate a smooth crossover from a mostly confined to a mostly deconfined phase, accompanied by a restoration of chiral symmetry [4,5]. The crossover region sharpens for increasing quark masses and turns in a second-order phase transition for critical values of the quarks masses, above which the transition is first order. The same is expected to happen with the chiral transition in the opposite limit of decreasing quark masses: the transition turns first order below a critical value of the quark masses. Although not firmly established by lattice calculations yet [6], this is the expected behavior of a theory with at least three light quark flavors. The situation with

Published by the American Physical Society under the terms of the Creative Commons Attribution 4.0 International license. Further distribution of this work must maintain attribution to the author(s) and the published article's title, journal citation, and DOI. Funded by SCOAP ${ }^{3}$. two light quarks is more subtle due to the possible role of the axial anomaly [7].

The situation is even less clear at $\mu_{B} \neq 0$ in the low quark mass region (including the physical point), where standard Monte Carlo algorithms are plagued by the infamous sign problem [8]. The typical expectation is that of a line of firstorder chiral transition at low temperatures ending at a critical point [9]. Firmly establishing the existence of the latter and studying its possible experimental signatures has been the topic of intense theoretical work [10-16] and is among the major physics goals of various present and upcoming experiments [17-20]. Methods to circumvent the sign problem on the lattice have been devised but remain, so far, limited to $\mu_{B} / T \lesssim 1$, and no critical end point (CEP) has been firmly established [21]. One strategy to go beyond is to employ approximate nonperturbative continuum approaches, based on Dyson-Schwinger (DSE) or functional renormalization group (FRG) equations, with increasing level of refinement [22-25]. These typically find a CEP at a relatively large $\mu_{B} / T \gtrsim 3$, where systematic errors are not completely under control [25]. A complementary approach uses phenomenological, Nambu-JonaLasinio (NJL) or quark-meson models with various degrees of sophistication [26-30]. These typically predict a CEP at relatively large $\mu_{B} / T$, whose precise location, however, varies significantly from one study to another. One common weakness of such approaches is that the employed approximations lack a systematic ordering principle. One typically explores the whole phase diagram with truncations adjusted against lattice data at $\mu_{B}=0$, far from the region where a critical point is found. 
In the present article, we undertake a study of this issue using a recently proposed semiperturbative approach to the infrared dynamics of QCD. This is based on the CurciFerrari (CF) model [31,32], a simple massive extension of the Faddeev-Popov (FP) Lagrangian in the Landau gauge, where the gluon mass term is motivated both by the results of lattice simulations [33] and by the necessity to modify the FP Lagrangian in the infrared due to Gribov ambiguities [34]. The CF model is the simplest renormalizable deformation of the FP Lagrangian and remains under perturbative control down to the deep infrared: The gluon mass screens the standard perturbative Landau pole and the (running) gauge coupling remains moderate at all scales $[32,35]$, as observed in lattice simulations. A series of recent studies has shown that the perturbative CF model gives an accurate description of the phase structure of pure Yang-Mills theories and of QCD with heavy quarks [36-38]. The case of light quarks is more delicate because, unlike the couplings in the pure gauge sector, the quarkgluon coupling becomes significant in the infrared [39]. A systematic approximation scheme, nonperturbative in the quark-gluon vertex, has been proposed in Ref. [40], based on a double expansion in powers of the pure gauge coupling and of the inverse number of colors $1 / N_{c}$. At leading order, this leads to the well-known rainbow equation with a tree-level structure for the (massive) onegluon exchange. Higher-order corrections being controlled by small parameters, one can treat renormalization group effects in a consistent way, which permits a systematic control of the ultraviolet momentum tails. The leadingorder approximation in this rainbow improved-loop expansion has been successfully implemented to describe the dynamics of chiral symmetry breaking in the vacuum in Ref. [40].

It is of definite interest to extend this approach to nonzero temperature and chemical potential and to study the phase diagram of the CF model in this expansion scheme. The present work aims at paving the way for such a systematic investigation. In particular, before attempting a full-fledged numerical solution of the relevant rainbow equation for the in-medium quark propagator, we wish to analyze the main qualitative features of the leading-order approximation. For instance, it is interesting to have an idea of the influence of the CF mass term on the phase structure of the theory and, also, to investigate the typical ballpark predictions of the model as compared to other approaches. In this spirit, we study here a simplified version of the inmedium rainbow equations, where we only retain the essential aspects of the physics of chiral symmetry with as much analytical control as possible.

In Sec. II, we briefly review the CF model and we derive the leading-order, rainbow equations for the in-medium quark propagator. We present two simplified versions of these equations in Sec. III, which use a form of localization in momentum space. Our results are detailed in Sec. IV and we conclude in Sec. V. Some technical material is gathered in the appendixes.

\section{THE RAINBOW EQUATION IN THE CURCI-FERRARI MODEL}

For simplicity, we study a theory with $N_{f}$ degenerate quark flavors. At nonzero temperature $T$ and quark chemical potential $\mu=\mu_{B} / 3$, the rainbow equation for the (Euclidean) quark propagator $S$ reads

$$
\begin{aligned}
S^{-1}(P)= & M_{0}-\left(i \hat{\omega}_{p}-\mu\right) \gamma_{0}-i \vec{p} \cdot \vec{\gamma} \\
& +g_{0}^{2} \int_{\hat{Q}}^{T} \gamma_{\mu} S(Q) \gamma_{\nu} G_{\mu \nu}(K),
\end{aligned}
$$

where $M_{0}$ and $g_{0}$ denote the bare quark mass and quarkgluon coupling, respectively. We have introduced Euclidean momenta $P=\left(\hat{\omega}_{p}, \vec{p}\right), Q=\left(\hat{\omega}_{q}, \vec{q}\right)$, and $K \equiv P-Q=$ $\left(\omega_{k}, \vec{k}\right)$, with $\omega_{n}=2 \pi n T$ and $\hat{\omega}_{n}=2 \pi(n+1 / 2) T$ $(n \in \mathbb{Z}$ ) being the bosonic and fermionic Matsubara frequencies, respectively. Correspondingly, the bosonic and fermionic Matsubara sums are denoted

$$
\begin{gathered}
\int_{K}^{T} f(K) \equiv T \sum_{k \in \mathbb{Z}} \int \frac{d^{3} k}{(2 \pi)^{3}} f\left(\omega_{k}, \vec{k}\right), \\
\int_{\hat{Q}}^{T} f(Q) \equiv T \sum_{q \in \mathbb{Z}} \int \frac{d^{3} q}{(2 \pi)^{3}} f\left(\hat{\omega}_{q}, \vec{q}\right),
\end{gathered}
$$

where it is implicitly understood that integrals over the norm of three-dimensional momenta are cut off at a scale $\Lambda$. The matrices $\gamma_{\mu}$ stand for the Euclidean Dirac matrices, with $\left\{\gamma_{\mu}, \gamma_{\nu}\right\}=2 \delta_{\mu \nu}$, which we choose in the Weyl basis, such that $\gamma_{0,2}^{*}=\gamma_{0,2}^{t}=\gamma_{0,2}$ and $\gamma_{1,3}^{*}=\gamma_{1,3}^{t}=-\gamma_{1,3}$. Finally, the tree-level gluon propagator is

$$
G_{\mu \nu}(K)=\frac{P_{\mu \nu}^{\perp}(K)}{K^{2}+m^{2}},
$$

with $P_{\mu \nu}^{\perp}(K)=\delta_{\mu \nu}-K_{\mu} K_{\mu} / K^{2}$ being the transverse projector and $m$ the CF mass.

In Appendix A, see also [13], we recall that the quark propagator decomposes as (we keep the $\mu$-dependence explicit)

$S\left(\hat{\omega}_{p}, \vec{p} ; \mu\right)=\tilde{B}+\left(i \hat{\omega}_{p}-\mu\right) \gamma_{0} \tilde{A}_{0}+i \vec{p} \cdot \vec{\gamma} \tilde{A}_{v}+i \gamma_{0} \vec{p} \cdot \vec{\gamma} \tilde{C}$,

with any of the components $\tilde{X}=\tilde{A}_{0}, \tilde{A}_{v}, \tilde{B}$ or $\tilde{C}$ depending on $\vec{p}$ only through its norm $p \equiv|\vec{p}|$ and such that

$$
\begin{aligned}
& \tilde{X}\left(-\hat{\omega}_{p}, p ;-\mu\right)=\tilde{X}\left(\hat{\omega}_{p}, p ; \mu\right), \\
& \tilde{X}\left(-\hat{\omega}_{p}, p ; \mu^{*}\right)^{*}=\tilde{X}\left(\hat{\omega}_{p}, p ; \mu\right) .
\end{aligned}
$$


These considerations apply also to the inverse propagator $S^{-1}\left(\hat{\omega}_{p}, \vec{p} ; \mu\right)$, which we parametrize as

$$
S^{-1}\left(\hat{\omega}_{p}, \vec{p} ; \mu\right)=B-\left(i \hat{\omega}_{p}-\mu\right) \gamma_{0} A_{0}-i \vec{p} \cdot \vec{\gamma} A_{v}-i \gamma_{0} \vec{p} \cdot \vec{\gamma} C .
$$

We have $X=\tilde{X} \Delta$, with

$$
\Delta=B^{2}+\left(\hat{\omega}_{p}+i \mu\right)^{2} A_{0}^{2}+p^{2}\left(A_{v}^{2}-C^{2}\right) .
$$

Projecting Eq. (1) onto the various tensor components, one arrives at the nonlinear system of integral equations

$$
\begin{aligned}
& B(P)=M_{0}+3 g_{0}^{2} C_{F} \int_{\hat{Q}}^{T} \frac{B(Q)}{\Delta(Q)} \frac{1}{K^{2}+m^{2}} \\
\hat{A}_{0}(P)= & \hat{\omega}_{p}+i \mu+g_{0}^{2} C_{F} \int_{\hat{Q}}^{T} \frac{1}{\Delta(Q)} \frac{1}{K^{2}+m^{2}} \\
\times & \left\{\hat{A}_{0}(Q)\left(1+2 \frac{\omega_{k}^{2}}{K^{2}}\right)+2 \hat{A}_{v}(Q) \frac{\omega_{k}}{K^{2}} \vec{k} \cdot \hat{q}\right\} \\
\hat{A}_{v}(P)= & p+g_{0}^{2} C_{F} \int_{\hat{Q}}^{T} \frac{1}{\Delta(Q)} \frac{1}{K^{2}+m^{2}} \\
& \times\left\{2 \hat{A}_{0}(Q) \frac{\omega_{k}}{K^{2}} \vec{k} \cdot \hat{p}+\hat{A}_{v}(Q)\right. \\
& \left.\times\left(\hat{p} \cdot \hat{q}+2 \frac{(\hat{p} \cdot \vec{k})(\vec{k} \cdot \hat{q})}{K^{2}}\right)\right\} \\
\hat{C}(P)= & g_{0}^{2} C_{F} \int_{\hat{Q}}^{T} \frac{\hat{C}(Q)}{\Delta(Q)} \frac{1}{K^{2}+m^{2}} \\
\times & \left\{\hat{p} \cdot \hat{q}\left(1-2 \frac{\omega_{k}^{2}}{K^{2}}-2 \frac{(\hat{p} \cdot \vec{k})(\vec{k} \cdot \hat{q})}{K^{2}}\right)\right\}
\end{aligned}
$$

where $\hat{A}_{0}(P) \equiv\left(\hat{\omega}_{p}+i \mu\right) A_{0}(P), \quad \hat{A}_{v}(P) \equiv p A_{v}(P)$ and $\hat{C}(P) \equiv p C(P)$, as well as $\hat{p} \equiv \vec{p} / p$. We have also introduced the quadratic Casimir in the fundamental representation $C_{F}=4 / 3$.

\section{LOCALIZATION}

As emphasized in the introduction, we do not attempt a direct solution of the above equations in the present article. In preparation of such a calculation, it is of interest to gain some insight into the main qualitative expectations concerning the phase diagram of the CF model. To this aim, we reduce the problem to its core technical simplicity, retaining only the essential aspects of the physics at hand.

In the chiral limit, corresponding to $M_{0} \rightarrow 0$, an unbroken chiral symmetry implies $B=C=0$, which obviously solves the (homogeneous) equations (10) and (13). Conversely, a solution with either $B \neq 0$ or $C \neq 0$ signals the spontaneous breaking of chiral symmetry. In what follows, we use $B$ as our order parameter for chiral symmetry breaking since $C=0$ remains an allowed solution (which we stick to) also away from the chiral limit. In order to keep the discussion as simple as possible, we also set the other functions to their tree-level values, $A_{0}=1, A_{v}=1$.

With this ansatz, the rainbow equation for the quark mass function $B$, Eq. (10), reads

$B(P)=M_{0}+4 g_{0}^{2} \int_{\hat{Q}}^{T} \frac{B(Q)}{Q_{i \mu}^{2}+B^{2}(Q)} \frac{1}{(P-Q)^{2}+m^{2}}$,

where we have defined $Q_{i \mu} \equiv\left(\hat{\omega}_{q}+i \mu, \vec{q}\right)$ and we recall that, in the case of a real chemical potential,

$$
B^{*}\left(\hat{\omega}_{p}, p ; \mu\right)=B\left(-\hat{\omega}_{p}, p ; \mu\right)=B\left(\hat{\omega}_{p}, p ;-\mu\right),
$$

as follows from Eqs. (6) and (7). In particular, $B$ is real for $\mu=0$ but becomes a priori complex when $\mu$ is nonzero. ${ }^{1}$

Our next level of simplification is to devise a versatile formulation of the problem that is both easily testable and allows for a maximum amount of analytic control. We use an approximation scheme called localization [41,42], which we now recall and extend to the problem at hand. The integral equation (14) couples the mass function at a given momentum to itself at all other momenta. However, there can be cases where, in some range of parameters and to a reasonable level of accuracy, the value of the mass function at a particular scale decouples from the rest and obeys, therefore, a simpler, "localized" equation.

For instance, in Refs. [41,42], such a localization procedure has been tested in the context of $\mathrm{O}(N)$ scalar field theories at nonzero temperature. In that case, the behavior of the mass function at zero momentum was essentially controlled by the zero-momentum mass itself and a self-consistent equation for this zero-momentum mode could be obtained by expanding the mass function about this zero-momentum value in the corresponding integrals. The solution of the localized equations was compared to actual solutions of the DSE equations for the propagators obtained from various truncations of the two-particle-irreducible effective action. In all the cases studied there, the local approximation gave very good (two-loop) to qualitatively good (three-loop) results as compared to a full numerical solution. We also mention that, even in the absence of a clear argument of why a certain scale should decouple, the localized equations often provide a good qualitative guide. In the present case, for

\footnotetext{
${ }^{1}$ An interesting exception that we exploit below is the zerotemperature limit for fixed integer $p$ in $\hat{\omega}_{p}$. In this limit, $\hat{\omega}_{p} \rightarrow 0$ and $B$ becomes real. We mention that it is also real in the case of an imaginary chemical potential.
} 
instance, it correctly captures the phenomenology of chiral symmetry breaking in the vacuum.

Here, we implement a similar localization procedure as that of Refs. [41,42], with the important difference that we deal here with fermion fields, which have no zero mode at nonzero temperature. In what follows, we propose two localization procedures to circumvent this issue, which also allow us to test the robustness of the approach by comparing the results in these two schemes.

Finally, we mention that the localization procedures proposed here can also serve for future studies of the rainbow equations in more intricate settings, such as the rainbow equation in the presence of nontrivial gluonic background (accounting for the interplay between chiral and center symmetry), or the corrections beyond the rainbow approximation, within the systematic expansion scheme alluded to in the introduction.

\section{A. Euclidean localization}

At finite temperature, it is important to stress that the Euclidean mass function $B$ is defined a priori on the fermionic Matsubara frequencies, which never vanish. We therefore localize the mass function at the smallest momentum available, that is, $B\left(\hat{\omega}_{1}\right) \equiv B\left(\hat{\omega}_{1}, 0 ; \mu\right)$. Since $B\left(\hat{\omega}_{1}\right)=B\left(-\hat{\omega}_{1}\right)^{*}$, it is natural-and in fact crucial, as we illustrate below-to localize $B\left(\hat{\omega}_{1}\right)$ together with $B\left(-\hat{\omega}_{1}\right)$. This means that we should consider two regions in the integrals, the one where it makes sense to expand the mass function about $\hat{\omega}_{1}$ and the one where it makes sense to expand it about $-\hat{\omega}_{1}$. Therefore, we approximate (14), with $P=\left(\hat{\omega}_{1}, \overrightarrow{0}\right)$ or $P=\left(-\hat{\omega}_{1}, \overrightarrow{0}\right)$ by

$$
\begin{aligned}
B\left(\hat{\omega}_{1}\right)= & M_{0}+2 g_{0}^{2} \int_{\hat{Q}}^{T} \frac{1}{\left(\hat{\omega}_{1}-\hat{\omega}_{q}\right)^{2}+q^{2}+m^{2}} \\
& \times\left[\frac{B\left(\hat{\omega}_{1}\right)}{\left(\hat{\omega}_{q}+i \mu\right)^{2}+q^{2}+B\left(\hat{\omega}_{1}\right)^{2}}\right. \\
& \left.+\frac{B\left(-\hat{\omega}_{1}\right)}{\left(\hat{\omega}_{q}+i \mu\right)^{2}+q^{2}+B\left(-\hat{\omega}_{1}\right)^{2}}\right],
\end{aligned}
$$

and

$$
\begin{aligned}
B\left(-\hat{\omega}_{1}\right)= & M_{0}+2 g_{0}^{2} \int_{\hat{Q}}^{T} \frac{1}{\left(\hat{\omega}_{1}+\hat{\omega}_{q}\right)^{2}+q^{2}+m^{2}} \\
& \times\left[\frac{B\left(\hat{\omega}_{1}\right)}{\left(\hat{\omega}_{q}+i \mu\right)^{2}+q^{2}+B\left(\hat{\omega}_{1}\right)^{2}}\right. \\
& \left.+\frac{B\left(-\hat{\omega}_{1}\right)}{\left(\hat{\omega}_{q}+i \mu\right)^{2}+q^{2}+B\left(-\hat{\omega}_{1}\right)^{2}}\right],
\end{aligned}
$$

which are easily checked to be compatible with $B\left(\hat{\omega}_{1}\right)=B\left(-\hat{\omega}_{1}\right)^{*}$. It is convenient to work with the real quantities

$$
\begin{gathered}
B_{r} \equiv \frac{B\left(\hat{\omega}_{1}\right)+B\left(-\hat{\omega}_{1}\right)}{2}, \\
B_{i} \equiv \frac{B\left(\hat{\omega}_{1}\right)-B\left(-\hat{\omega}_{1}\right)}{2 i} .
\end{gathered}
$$

In terms of $B_{r}$ and $B_{i}$, the rainbow equations for $B\left( \pm \hat{\omega}_{1}\right)$ read

$$
\begin{aligned}
B_{r}= & M_{0}+2 g_{0}^{2} \int_{Q}^{T} \frac{1}{\left(\hat{\omega}_{1}-\hat{\omega}_{q}\right)^{2}+q^{2}+m^{2}} \\
& \times \operatorname{Re}\left[\frac{B_{r}+i B_{i}}{\left(\hat{\omega}_{q}+i \mu\right)^{2}+q^{2}+\left(B_{r}+i B_{i}\right)^{2}}\right. \\
& \left.+\frac{B_{r}-i B_{i}}{\left(\hat{\omega}_{q}+i \mu\right)^{2}+q^{2}+\left(B_{r}-i B_{i}\right)^{2}}\right],
\end{aligned}
$$

and

$$
\begin{aligned}
B_{i}= & 2 g_{0}^{2} \int_{Q}^{T} \frac{1}{\left(\hat{\omega}_{1}-\hat{\omega}_{q}\right)^{2}+q^{2}+m^{2}} \\
& \times \operatorname{Im}\left[\frac{B_{r}+i B_{i}}{\left(\hat{\omega}_{q}+i \mu\right)^{2}+q^{2}+\left(B_{r}+i B_{i}\right)^{2}}\right. \\
& \left.+\frac{B_{r}-i B_{i}}{\left(\hat{\omega}_{q}+i \mu\right)^{2}+q^{2}+\left(B_{r}-i B_{i}\right)^{2}}\right] .
\end{aligned}
$$

After performing the Matsubara sums and the angular integrals, Eqs. (20) and (21) rewrite in the simple form

$$
\begin{aligned}
M_{0}= & B_{r}-\frac{g_{0}^{2}}{2 \pi^{2}} \operatorname{Re}\left[\left(B_{r}+i B_{i}\right) F\left(B_{r}+i B_{i}\right)\right. \\
& \left.+\left(B_{r}-i B_{i}\right) F\left(B_{r}-i B_{i}\right)\right], \\
0= & B_{i}-\frac{g_{0}^{2}}{2 \pi^{2}} \operatorname{Im}\left[\left(B_{r}+i B_{i}\right) F\left(B_{r}+i B_{i}\right)\right. \\
& \left.+\left(B_{r}-i B_{i}\right) F\left(B_{r}-i B_{i}\right)\right],
\end{aligned}
$$

where, for notational convenience, we have defined

$$
F(B) \equiv F_{\mathrm{vac}}(B)+F_{\mathrm{th}}(B),
$$

with

$$
F_{\mathrm{vac}}(B) \equiv \int_{0}^{\Lambda} \mathrm{d} q \frac{q^{2}}{\varepsilon_{q}^{m} \varepsilon_{q}^{B}} \frac{1}{\varepsilon_{q}^{B}+\varepsilon_{q}^{m}}
$$




$$
\begin{aligned}
F_{\mathrm{th}}(B) \equiv & \int_{0}^{\Lambda} \mathrm{d} q \frac{q^{2}}{\varepsilon_{q}^{m} \varepsilon_{q}^{B}}\left\{\frac{\left(\mu-i \hat{\omega}_{1}\right)^{2} /\left(\varepsilon_{q}^{B}+\varepsilon_{q}^{m}\right)}{\left(\varepsilon_{q}^{B}+\varepsilon_{q}^{m}\right)^{2}-\left(\mu-i \hat{\omega}_{1}\right)^{2}}\right. \\
& +\frac{\varepsilon_{q}^{B} n_{\varepsilon_{q}^{m}}^{(-)}}{\left(\varepsilon_{q}^{B}\right)^{2}-\left(\varepsilon_{q}^{m}-\mu+i \hat{\omega}_{1}\right)^{2}} \\
& +\frac{\varepsilon_{q}^{B} n_{\varepsilon_{q}^{m}}^{(-)}}{\left(\varepsilon_{q}^{B}\right)^{2}-\left(\varepsilon_{q}^{m}+\mu-i \hat{\omega}_{1}\right)^{2}} \\
& -\frac{\varepsilon_{q}^{m} n_{\varepsilon_{q}^{B}-\mu}^{(+)}}{\left(\varepsilon_{q}^{m}\right)^{2}-\left(\varepsilon_{q}^{B}-\mu+i \hat{\omega}_{1}\right)^{2}} \\
& \left.-\frac{\varepsilon_{q}^{m} n_{\varepsilon_{q}^{B}+\mu}^{(+)}}{\left(\varepsilon_{q}^{m}\right)^{2}-\left(\varepsilon_{q}^{B}+\mu-i \hat{\omega}_{1}\right)^{2}}\right\},
\end{aligned}
$$

$$
M_{0}=\left(1-\frac{g_{0}^{2}}{\pi^{2}}\left[F_{\mathrm{vac}}(B)+F_{\mathrm{th}}(B)\right]\right) B,
$$

where $F(B)$ is to be evaluated with $\hat{\omega}_{1} \rightarrow 0$ and $\mu \rightarrow 0$ everywhere but in the Fermi-Dirac factors $n_{\varepsilon_{q}^{B} \pm \mu}^{(+)}$. While $F_{\text {vac }}(B)$ remains the same as above, we now have

$$
F_{\mathrm{th}}(B)=\frac{2}{B^{2}-m^{2}} \int_{0}^{\infty} d q q^{2}\left(\frac{n_{\varepsilon_{q}^{m}}^{(-)}}{\varepsilon_{q}^{m}}+\frac{n_{\varepsilon_{q}^{B}-\mu}^{(+)}+n_{\varepsilon_{q}^{B}+\mu}^{(+)}}{2 \varepsilon_{q}^{B}}\right) .
$$

with $\varepsilon_{y}^{x} \equiv \sqrt{x^{2}+y^{2}}$ and where $n_{x}^{( \pm)}=\left(e^{x} \pm 1\right)^{-1}$ denote the Bose-Einstein and Fermi-Dirac distributions. We note that $F\left(B^{*}\right)$ is not the complex conjugate of $F(B)$, due to the dependence on $i \hat{\omega}_{1}$, which we leave, however, implicit in what follows.

\section{B. Physical localization}

One inconvenient aspect of the Euclidean localization is that one has to deal with two variables, $B_{r}$ and $B_{i}$. This prevents the definition of a potential associated to the localized equations, due to the fact that the latter do not comply with the Schwarz conditions. Although many features of the phase diagram do not require the existence of an underlying potential, it is convenient to find a setting where one deals with only one variable instead of two. ${ }^{2}$ Another drawback of the Euclidean localization is that it involves the first Matsubara frequency $\pi T$. Therefore, we expect its quality to decrease as the temperature is increased.

One way to cope with these limitations is to consider a localization based on the retarded mass function

$$
B_{R}\left(p_{0}, p\right)=B\left(\hat{\omega}_{p} \rightarrow-i\left(p_{0}+\mu\right)+0^{+}, p\right),
$$

evaluated for $p_{0}=0$ and $p=0$. The reason for the presence of $\mu$ in the prescription to obtain the physical retarded Green's function is recalled in Appendix B; see also Ref. [43]. We find that, for $B$ real and smaller than $m$, the corresponding analytic continuation of $F(B)$ in Eq. (24) is real in the limit $p_{0} \rightarrow 0 .^{3}$ Therefore, the equation for $B_{i}$ becomes compatible with the solution $B_{i}=0$, which we assume from now on, and the equation for $B_{r} \equiv B$ reduces down to

\footnotetext{
${ }^{2}$ We see below that, after renormalization, there is one particular way to achieve this within the Euclidean localization. There are also certain limits, such as $T \rightarrow 0$ or $\mu \rightarrow 0$, where this becomes possible due to the fact that $B_{i} \rightarrow 0$.

${ }^{3}$ One should pay attention to the fact that what is really continued are not $B_{r}$ and $B_{i}$, but rather $B(\omega)$.
}

One price to pay for this choice of localization is the singularity at $B=m$, which is regulated in the retarded self-energy through $B^{2}-m^{2} \rightarrow B^{2}-m^{2}+i 0^{+}$. We ignore this issue and restrict our analysis to cases where $B<m$. This is justified both because the physically relevant values in the vacuum fall in this range (see below) and because most of our discussions below concern the vicinity of the symmetric point $B=0 .{ }^{4}$ We note finally that, interestingly (although not surprisingly), the localized equation (28) reduces to the corresponding mean-field gap equation of a NJL-type model with an effective nonlocal four-fermion vertex corresponding to a massive gluon exchange.

\section{RESULTS}

We now investigate our predictions for the phase diagram using the two types of localizations, starting with the physical localization.

\section{A. Physical localization}

Consider first the chiral limit, corresponding to $M_{0} \rightarrow 0$. For large enough values of the coupling, Eq. (28) admits nontrivial, symmetry-breaking solutions on top of the chirally symmetric solution $B=0$. It is convenient to parametrize the equation in terms of the dynamical mass in the vacuum, $B_{0} \equiv B(T=0, \mu=0)$, given by

$$
F_{\text {vac }}\left(B_{0}\right)=\pi^{2} / g_{0}^{2},
$$

where, because $F_{\text {vac }}(B)$ is a decreasing function of $B$, we see that symmetry-breaking solutions exist only if $g_{0}^{2}>\pi^{2} / F_{\text {vac }}(0)$. We use Eq. (30) to trade the bare quark-gluon coupling $g_{0}$ for the (ultraviolet finite) quark mass $B_{0}$. The rainbow equation rewrites as

$0=B\left[F_{\mathrm{vac}}\left(B_{0}\right)-F_{\mathrm{vac}}(B)-F_{\mathrm{th}}(B)\right] \equiv 2 B R\left(B^{2}\right)$,

where we note that the cutoff can now be sent to infinity,

\footnotetext{
${ }^{4}$ On the other hand, the constraint $B<m$ does not allow for a smooth continuation from the chiral limit to the heavy quark limit since $B$ is always constrained to be less than $m$. This is one of the reasons why we also develop the Euclidean localization.
} 


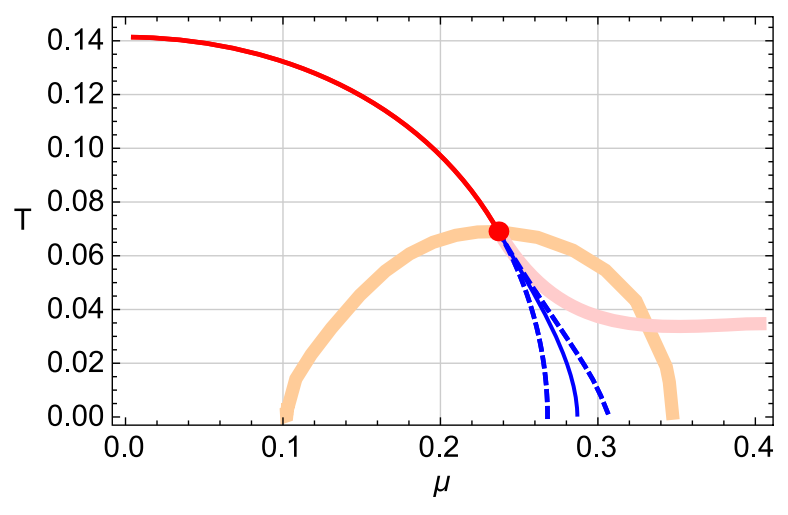

FIG. 1. Phase diagram in the chiral limit (all scales in $\mathrm{GeV}$ ), as obtained from the physical localization of the rainbow equation, with $B_{0}=0.3 \mathrm{GeV}$ and $m=0.5 \mathrm{GeV}$. The line of second-order transitions (solid red) turns into a line of first-order transitions (solid blue) at the tricritical point (red dot). The dashed curves are the corresponding spinodal lines. The orange line shows the location of the tricritical point as a function of the gluon mass (as described in the main text). Away from the chiral limit, the tricritical point turns into a CEP, whose position follows the pink line as the bare quark mass is increased.

$F_{\mathrm{vac}}\left(B_{0}\right)-F_{\mathrm{vac}}(B)=\frac{B^{2} \ln (B / m)}{2\left(B^{2}-m^{2}\right)}-\frac{B_{0}^{2} \ln \left(B_{0} / m\right)}{2\left(B_{0}^{2}-m^{2}\right)}$.

It is useful to interpret Eq. (31) as deriving from a chirally symmetric potential $W\left(B^{2}\right)$, with $\partial_{B} W\left(B^{2}\right)=$ $2 B R\left(B^{2}\right)$, that is $W^{\prime}\left(B^{2}\right)=R\left(B^{2}\right)$. The absolute minima of $W\left(B^{2}\right)$ then determine the state of the system. At $\mu=0$, one easily checks that the nontrivial minimum, equal to $B_{0}$ in the vacuum, decreases with increasing temperature and continuously reaches $B=0$ at a critical temperature. This extends in a critical line of second-order phase transitions $T_{c}(\mu)$ in the $(\mu, T)$ plane, defined by the condition $W^{\prime}(0)=0$, as shown in Fig. 1. Depending on the parameters, this critical line can turn into a line of first-order transitions at a tricritical point, defined by the conditions $\left.\partial_{B}^{(2 n)} W\left(B^{2}\right)\right|_{B=0}=0$ for $n=1,2$ or, equivalently,

$$
W^{\prime}(0)=W^{\prime \prime}(0)=0 .
$$

The first-order line is then determined from

$$
W^{\prime}\left(B_{\min }^{2}\right)=W\left(B_{\min }^{2}\right)-W(0)=0,
$$

where $B_{\min }$ is the nontrivial minimum at the transition. The associated lower and upper spinodals are respectively defined by

$$
W^{\prime}(0)=0 \quad \text { and } \quad W^{\prime}\left(B_{\mathrm{sp}}^{2}\right)=W^{\prime \prime}\left(B_{\mathrm{sp}}^{2}\right)=0,
$$

with $B_{\text {sp }}$ being the location of the nontrivial metastable state at the upper spinodal. The two spinodals flank the firstorder line and merge at the tricritical point, beyond which the lower spinodal becomes the critical line. The equation governing the critical and lower spinodal lines is easily obtained as

$\mu^{2}(T)=m^{2} \frac{B_{0}^{2} \ln \left(B_{0} / m\right)}{B_{0}^{2}-m^{2}}-4 \int_{0}^{\infty} d q q^{2} \frac{n_{\varepsilon_{q}^{m}}^{(-)}}{\varepsilon_{q}^{m}}-\frac{\pi^{2}}{3} T^{2}$,

which is a strictly concave line. For low enough temperatures $T / m \ll 1$, the gluonic thermal contribution (second term) in Eq. (36) is negligible and one obtains the approximate expression

$$
\mu^{2}(T) \approx m^{2} \frac{B_{0}^{2} \ln \left(B_{0} / m\right)}{B_{0}^{2}-m^{2}}-\frac{\pi^{2}}{3} T^{2} .
$$

This is similar to the result obtained in the quark-meson model with a large- $N_{f}$ approximation [26].

We note that there is an ambiguity in the definition of the potential $W\left(B^{2}\right)$ since neither the solutions of Eq. (31) nor their convexity are altered by the replacement $W^{\prime}\left(B^{2}\right) \rightarrow$ $f_{+}\left(B^{2}\right) R\left(B^{2}\right)$ with $f_{+}\left(B^{2}\right)$ being a differentiable and strictly positive function. Interestingly, because the conditions (33) and (35) only involve $W^{\prime}$ and its derivatives, they are, in fact, independent of the function $f_{+}$and so are, thus, the spinodal lines, the line of second-order transition, and, of course, the tricritical point where these lines meet. Only the line of first-order transition explicitly depends on $f_{+}$through the second condition in Eq. (34). However, it always lies in between the two spinodals.

In Fig. 1, we show our results for the phase diagram in the chiral limit. We use the typical values $m=500 \mathrm{MeV}$ and $B_{0}=300 \mathrm{MeV}$, motivated by the study of dynamical chiral symmetry breaking in the CF model in the vacuum [40]. We find a tricritical point located at $(\mu, T) \approx$ $(237 \mathrm{MeV}, 69 \mathrm{MeV})$. The transition at zero chemical potential occurs at $T_{c}(\mu=0) \approx 141 \mathrm{MeV}$ and the two spinodals meet the $T=0$ axis for $\mu \approx 268 \mathrm{MeV}$, and $\mu \approx 305 \mathrm{MeV}$, respectively. This gives an estimate of the first-order transition line at most at the $10 \%$ level, independently of the function $f_{+}$(the estimate improves as one approaches the tricritical point). For the choice $f_{+}=1$, the $T=0$ transition point is at $\mu \approx 287 \mathrm{MeV}$.

In fact, Eq. (31) greatly simplifies at $T=0$, where

$$
F_{\mathrm{th}}(B)=\frac{B^{2} / 2}{B^{2}-m^{2}}\left[\frac{\mu}{B} \sqrt{\frac{\mu^{2}}{B^{2}}-1}-\cosh ^{-1}\left(\frac{\mu}{B}\right)\right],
$$

if $\mu \geq B$ and $F_{\text {th }}(B)=0$ otherwise. In particular, we see that the value of the order parameter below the transition point is independent of $\mu, B(T=0, \mu)=B_{0}$, until it jumps to $B=0$ in the symmetric phase. This is known as the 


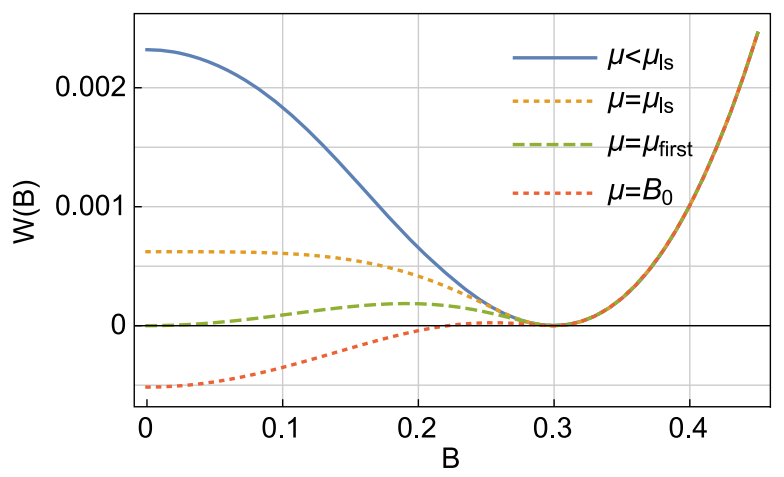

FIG. 2. Illustration of the silver-blaze property: as long as $\mu$ is below the first-order transition, the minimum of the potential sits at $B=B_{0}=0.3 \mathrm{GeV}$. For $\mu_{\text {first }}<\mu<B_{0}$, there is still an extremum at $B=B_{0}$, but it is not the absolute minimum anymore. For $\mu>B_{0}$, this local minimum moves away from $B_{0}$ and disappears at the upper spinodal $\mu_{u s}$ (slightly above $\mu=B_{0}$, not shown).

silver-blaze property $[44,45]$, which we illustrate in Fig. . $^{5}$ Also, using Eqs. (32) and (38), we can determine the values of the gluon mass for which there exists a tricritical point. The latter reaches the $T=0$ axis for some values of the ratio $x=m / B_{0}$. Defining $u=\left(\ln x^{2}\right) /\left(x^{2}-1\right)$, we get the condition $u=1+\ln (2 u)$, which has two solutions in $\mathbb{R}^{+}$, $u_{ \pm}=u\left(x_{ \pm}\right)$, with $x_{+} x_{-}=1$. One finds $x_{-}=\sqrt{u_{+} / u_{-}} \approx$ 0.294 and $x_{+} \approx 3.398$. The corresponding values of $y=$ $\mu / B_{0}$ are given by Eq. (36) at $T=0, y^{2}=x^{2} u / 2$, yielding $y_{-}=\sqrt{u_{+} / 2} \approx 0.340$ and $y_{+}=y_{-} / x_{-} \approx 1.157$. There exists a tricritical point iff $x \in\left[x_{-}, x_{+}\right]$as shown in Fig. 1.

Let us now move away from the chiral limit. Equation (31) now reads $2 B R\left(B^{2}\right)=H$, where $H \equiv$ $\pi^{2} M_{0} / g_{0}^{2}$ needs to be seen as a finite parameter controlling the departure from the chiral limit. For $H \neq 0$, the secondorder transitions turn into crossovers and the tricritical point becomes a CEP terminating a first-order line. Writing the potential $V(B)=-H B+W\left(B^{2}\right)$, the conditions for a critical point are

$$
V^{\prime}\left(B_{c}\right)=V^{\prime \prime}\left(B_{c}\right)=V^{\prime \prime \prime}\left(B_{c}\right)=0,
$$

from which one extracts $B_{c}, T_{c}$, and $\mu_{c}$ for each $H$. To this aim, it is convenient to vary $B$, and determine $T_{c}(B)$ and

\footnotetext{
${ }^{5}$ We mention that the silver-blaze property should, in principle, extend only up to the first singularity on the $T=0$ axis, namely, the nuclear liquid-gas transition. Here, however, our level of description does not capture the corresponding dynamics, and the silver-blaze property extends further. We also mention that the $\mu$-independence below the first singularity applies in principle only to 0 -point functions. For higher $n$-point functions, it takes a more general form as shown in [45]. However, due to the presence of $\mu$ in the retarded prescription (27), it can be argued that the silver-blaze property applies to retarded Green functions as it does for 0-point functions.
}

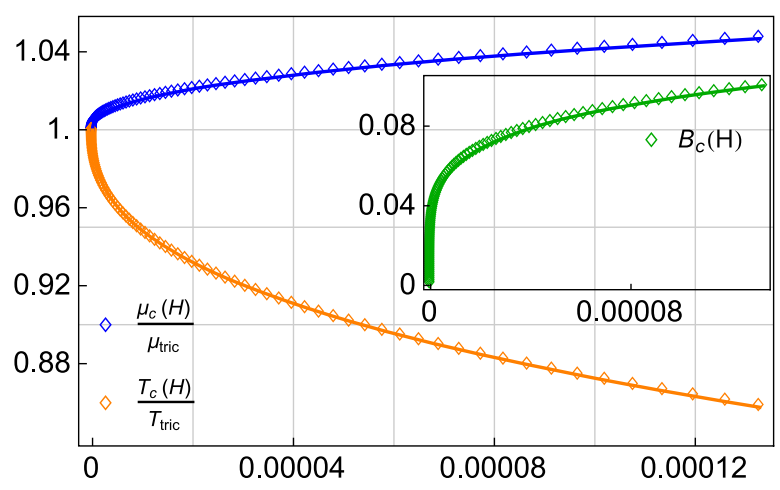

FIG. 3. The approach of the critical quantities $\mu_{c}(H), T_{c}(H)$, and $B_{c}(H)$ to the chiral limit shows mean-field tricritical scaling. The solid lines are power law fits of the form $A_{c}(H)-A_{\text {tric }} \propto$ $H^{\omega_{A}}$ for $A=\mu, T, B$ with mean-field exponents $\omega_{T}=\omega_{\mu}=2 / 5$ and $\omega_{B}=1 / 5$.

$\mu_{c}(B)$ from the last two conditions in Eq. (39) - that do not involve $H$-and then deduce $H(B)$ from the first condition. Inverting this relation, one then gains access to $T_{c}(H)$ and $\mu_{c}(H)$. In particular, we find that the approach to tricriticality is governed by mean-field exponents; see Fig. 3. This is expected because the potential is regular around $B=0$. The trajectory of the CEP in the phase diagram, shown in Fig. 1, exhibits a nonmonotonous behavior of $T_{c}$ as a function of $\mu$, similar to that observed in Ref. [12] using an approach based on the Cornwall-Jackiw-Tomboulis effective potential. Finally, Fig. 4 shows the interval $\left[x_{-}(H), x_{+}(H)\right]$ compatible with a critical point for each value of $H$. Interestingly, in the physical localization considered here, the CF mass should be neither too large nor too small for a CEP to exist.

\section{B. Euclidean localization}

Let us now investigate the Euclidean localization that allows us to test the robustness of the previous features.

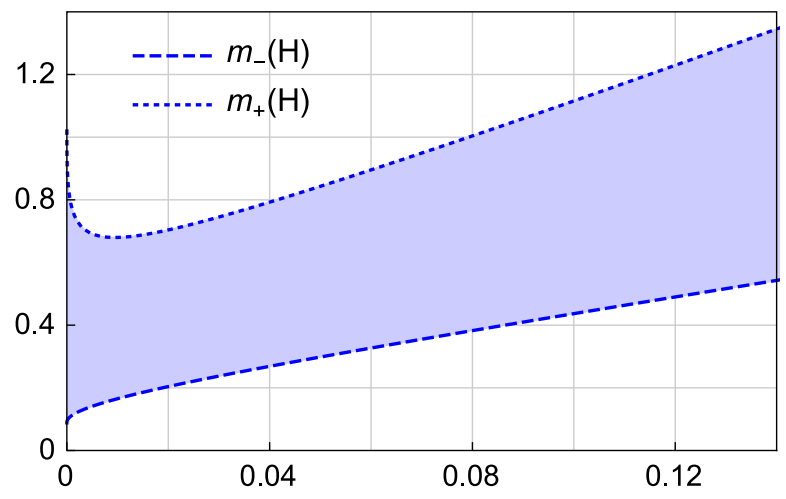

FIG. 4. Allowed values of the gluon mass $m$ for a (tri)critical point to exist as a function of $H$ (blue area). All scales are in $\mathrm{GeV}$ and $m_{ \pm} \equiv B_{0} x_{ \pm}$. 
To renormalize the equations in this case, we proceed as follows. First, we note that the function $F_{\text {vac }}(B)$ possesses a logarithmic UV divergence that however does not depend on $B$. It is then readily checked that the equation for $B_{r}$ has a divergence proportional to $B_{r}$. This divergence can be absorbed into a redefinition of the bare coupling as follows. We divide the corresponding equation by $g_{0}^{2}$ and set

$$
\frac{1}{g_{0}^{2}}=\frac{1}{g^{2}}+\frac{1}{\pi^{2}} F_{\mathrm{vac}}\left(B_{\star}\right),
$$

where the renormalized coupling $g$ should be interpreted as being defined at the (real) renormalization scale $B_{\star}$. Introducing $H \equiv \pi^{2} M_{0} / g_{0}^{2}$ as before, the renormalized equation for $B_{r}$ takes the form

$$
\begin{aligned}
H= & \frac{\pi^{2}}{g^{2}} B_{r}-\frac{1}{2} \operatorname{Re}\left[\left(B_{r}+i B_{i}\right) \tilde{F}\left(B_{r}+i B_{i}\right)\right. \\
& \left.+\left(B_{r}-i B_{i}\right) \tilde{F}\left(B_{r}-i B_{i}\right)\right],
\end{aligned}
$$

with $\tilde{F}(B)=F_{\text {vac }}(B)-F_{\text {vac }}\left(B_{\star}\right)+F_{\text {th }}(B)$.

As far as the equation for $B_{i}$ is concerned, it is easily checked that it is finite, for any fixed $g_{0}$. Therefore, using the redefinition (40) or the bare coupling is problematic here since it leads to a spurious cutoff dependence. This problem is rooted in the localization procedure that does not commute with the renormalization procedure; see [42] for more details. However, we can always define a renormalized localized scheme by replacing $g_{0}$ by $g$ in the equation for $B_{i}$,

$$
\begin{aligned}
0= & \frac{\pi^{2}}{g^{2}} B_{i}-\frac{1}{2} \operatorname{Im}\left[\left(B_{r}+i B_{i}\right) \tilde{F}\left(B_{r}+i B_{i}\right)\right. \\
& \left.+\left(B_{r}-i B_{i}\right) \tilde{F}\left(B_{r}-i B_{i}\right)\right]
\end{aligned}
$$

We note that, in this equation, one can interchangeably use $F$ or $\tilde{F}$.

With this choice of renormalization, we can eventually express the equations in terms of the vacuum mass in the chiral limit, $B_{0}$; see Eq. (30). It is related to $g$ and $B_{\star}$ by

$$
F_{\text {vac }}\left(B_{0}\right)-F_{\text {vac }}\left(B_{\star}\right)=\pi^{2} / g^{2} .
$$

Replacing $g^{2}$ in that form, one checks that the first term in the rhs of (41) disappears while the scale $B_{\star}$ in $\tilde{F}(B)$ is replaced by $B_{0}$. Thus, Eq. (41) does not depend on $B_{\star}$. However a scale dependence remains in Eq. (42), as expected at a given order of approximation. Below, we test the dependence of our results on the renormalization scale $B_{\star}$.

Let us also mention that, because of the localization procedure, the coupled gap equations (41) and (42), which we denote formally as $H=\mathcal{R}_{r}\left(B_{r}, B_{i}\right)$ and $0=\mathcal{R}_{i}\left(B_{r}, B_{i}\right)$ in what follows, cannot be seen as deriving from a potential, because, in general the Schwarz condition $\partial \mathcal{R}_{r} / \partial B_{i}=\partial \mathcal{R}_{i} / \partial B_{r}$ is not satisfied. However, certain features of the phase diagram can be defined without the need of a potential because they correspond to the merging of different solutions of the gap equations. For instance, suppose that we want to investigate whether $B_{r}$ becomes critical. To this purpose, we solve for $B_{i}$ as a function of $B_{r}$ from its gap equation,

$$
0=\mathcal{R}_{i}\left(B_{r}, B_{i}\left(B_{r}\right)\right)
$$

and construct a potential for $B_{r}$ by integrating

$$
V^{\prime}\left(B_{r}\right)=\mathcal{R}_{r}\left(B_{r}, B_{i}\left(B_{r}\right)\right) .
$$

In the chiral limit, the criticality condition reads $0=V^{\prime \prime}(0)$. Simple algebra using (44) and (45) leads to the condition

$$
0=\frac{\partial \mathcal{R}_{r}}{\partial B_{r}}-\left.\frac{\partial \mathcal{R}_{r}}{\partial B_{i}}\left(\frac{\partial \mathcal{R}_{i}}{\partial B_{i}}\right)^{-1} \frac{\partial \mathcal{R}_{i}}{\partial B_{r}}\right|_{B_{r}=B_{i}=0} .
$$

Now, it is easily verified that $\mathcal{R}_{r}\left(B_{r}, B_{i}\right)=\mathcal{R}_{r}\left(B_{r},-B_{i}\right)$, from which it follows that $\partial \mathcal{R}_{r} /\left.\partial B_{i}\right|_{B_{i}=0}=0$. Similarly, writing $\mathcal{R}_{i}\left(B_{r}, B_{i}\right) \equiv B_{i} \pi^{2} / g^{2}+\tilde{\mathcal{R}}_{i}\left(B_{r}, B_{i}\right)$, we have $\tilde{\mathcal{R}}_{i}\left(B_{r},-B_{i}\right)=\tilde{\mathcal{R}}_{i}\left(B_{r}, B_{i}\right)$ and therefore $\partial \mathcal{R}_{i} /\left.\partial B_{i}\right|_{B_{i}=0}=$ $\pi^{2} / g^{2}$. From these remarks, it follows that the condition for a critical point in the chiral limit simplifies to

$$
0=\left.\frac{\partial \mathcal{R}_{r}}{\partial B_{r}}\right|_{B_{r}=B_{i}=0} .
$$

The same equation defines the lower spinodal in the case of a first-order phase transition. The upper spinodal is also determined from (46) but without evaluating it for $B_{r}=B_{i}=0$ and coupling it to the gap equation for $B_{r}$. Finally, the tricritical point is determined from conditions $0=V^{\prime \prime}(0)=V^{(4)}(0)$. We find

$$
0=\left.\sum_{u, v, w} \frac{\partial^{3} \mathcal{R}_{r}}{\partial B_{u} \partial B_{v} \partial B_{w}}\right|_{B=0} \Delta B_{r} \Delta B_{u} \Delta B_{v} \Delta B_{w},
$$

where the indices $u, v$, and $w$ take the values $r$ or $i$, and $\Delta B=\left(\partial \mathcal{R}_{i} / \partial B_{i},-\partial \mathcal{R}_{i} / \partial B_{r}\right)_{B_{r}=B_{i}=0}$. We have again made use of $\partial \mathcal{R}_{r} /\left.\partial B_{i}\right|_{B_{i}=0}=0$. This formula simplifies further because $\partial^{3} \mathcal{R}_{r} /\left.\partial B_{r}^{2} \partial B_{i}\right|_{B_{i}=0}=\partial^{3} \mathcal{R}_{r} /\left.\partial B_{i}^{3}\right|_{B_{i}=0}=0$. We note however that we are not able to fully eliminate $B_{i}$, contrary to what happened for the critical point (see below for a particular limit where this becomes possible).

Our results in the chiral limit (with $B_{\star}=1 \mathrm{GeV}$ ) are summarized in Table I and compared to the results in the physical localization as well as to the results in other approaches. The last column shows the values of $\mu$ at which the lower and upper spinodals $\left(\mu_{\mathrm{ls}}\right.$ and $\left.\mu_{\mathrm{us}}\right)$ and the firstorder transition $\left(\mu_{\text {first }}\right)$ are reached for $T=0$. As already 
TABLE I. Results in the chiral limit for the two considered localizations, in comparison to benchmark literature findings. All values are given in $\mathrm{MeV}$. For the Euclidean localization, we have chosen a renormalization scale $B_{\star}=1 \mathrm{GeV}$.

\begin{tabular}{lcccccc}
\hline \hline Chiral limit $(H=0)$ & $\mu_{\text {tric }}$ & $T_{\text {tric }}$ & $T_{\mathrm{c}}$ & $\mu_{\mathrm{ls}}$ & $\mu_{\text {first }}$ & $\mu_{\mathrm{us}}$ \\
\hline Physical localization & 237 & 69 & 141 & 268 & 287 & 305 \\
Euclidean localization & 318 & 64 & 150 & 346 & 365 & 376 \\
Hatta et al. [12] & 209 & 107 & - & & & \\
Qin et al. [46] A & 140 & 110 & 124 & & & \\
Qin et al. [46] B & 130 & 120 & 133 & & & \\
Schaefer et al. [47] & 251 & 52 & 142 & & & \\
Jakovac et al. [26] & 280 & 60 & 140 & & & \\
Costa et al. [48] & 286 & 112 & 215 & & & \\
\hline \hline
\end{tabular}

discussed in the previous section, the value of $\mu_{\text {first }}$ has the largest uncertainty since it depends on the potential that is not uniquely defined in our approach. Also, it may look surprising that we could obtain a value $\mu_{\text {first }}$ in the Euclidean localization case since there is no potential in this case compatible with the gap equations. However, in the $T \rightarrow 0$ limit, it is readily checked using (15) that $B_{i}$ vanishes. We note also that, along the $T=0$ axis, $B \equiv B_{r}$ is not constant below the transition. This is of course not in contradiction with the silver-blaze property since only 0 -point functions should be constant. The case of the physical localization is a bit peculiar since the retarded prescription (27) with $\mu$ included makes the retarded function behave like a 0-point function as far as the silver-blaze property is concerned.

We mentioned above that it was crucial to localize simultaneously in $B\left(\hat{\omega}_{1}\right)$ and $B\left(-\hat{\omega}_{1}\right)$. Let us illustrate this point here. In Fig. 5, we show the curves $\mathcal{R}_{r}\left(B_{r}, B_{i}\right)=0$ and $\mathcal{R}_{i}\left(B_{r}, B_{i}\right)=0$ for decreasing temperatures and a large enough chemical potential. The crossings correspond to the various possible solutions in the chiral limit and, because the chemical potential is chosen large enough, we should observe a first-order transition pattern. Let us now see how this comes about. The first plot is at a temperature right above the upper spinodal, that is, the appearance of a new crossing between the curves $\mathcal{R}_{r}\left(B_{r}, B_{i}\right)=0$ and $\mathcal{R}_{i}\left(B_{r}, B_{i}\right)=0$, at which two new extrema are about to appear. ${ }^{6}$ Below this temperature, the various branches making the curve $\mathcal{R}_{r}\left(B_{r}, B_{i}\right)=0$ fuse and reorganize, in such a way that, at an even lower temperature, a second spinodal occurs at $B=0$ where two extrema merge. We observe that the proper realization of this scenario requires not only the various branches to fuse at some temperatures, but also that the number of intersections of the curves $\mathcal{R}_{r}\left(B_{r}, B_{i}\right)=0$ and $\mathcal{R}_{i}\left(B_{r}, B_{i}\right)=0$ changes from 1 to 5 , and then to 3 , as the temperature is decreased. Had we

\footnotetext{
${ }^{6}$ In fact, there are four such extrema since, in the chiral limit, the problem is symmetric under $\left(B_{r}, B_{i}\right) \rightarrow\left(-B_{r},-B_{i}\right)$. But it is then enough to restrict to $B_{r}>0$.
}

performed the localization only with respect to $B\left(\hat{\omega}_{1}\right)$, that is by writing only the first term in Eq. (16) and taking the real and imaginary part of the corresponding equation, this second requirement would not be fulfilled, as we have checked explicitly.

Regarding the renormalization scale dependence of our results, we observe numerically that the critical/lower spinodal line does not depend on the scale $B_{\star}$. This is no surprise since the corresponding equation (46) depends only on $\mathcal{R}_{r}\left(B_{r}, 0\right)$, which, as we have already argued above is $B_{\star}$ independent. In contrast, the position of the tricritical point along (46), or even the other spinodal emerging from this point, does depend on $B_{\star}$. We note, however, that the inverse coupling $1 / g^{2}$ diverges positively as the renormalization scale is taken to infinity; see Eq. (43). Since there is no other dependence with respect to $B_{\star}$ in Eq. (42), it follows that $B_{i}$ should approach 0 in this limit and all relevant features (boundary lines, tri/ critical points, ...) should converge to a certain limit, obtained by considering a single gap equation (41) in which one sets $B_{i}=0$ from the start. ${ }^{7}$ Take for instance the tricritical point. Because $\Delta B_{r}=-\pi^{2} / g^{2} \gg \Delta B_{i}$ in the limit $B_{\star} \rightarrow \infty$, Eq. (48) becomes

$$
0=\left.\frac{\partial^{3} \mathcal{R}_{r}}{\partial B_{r}^{3}}\right|_{B=0},
$$

which is indeed the condition for a tricritical point if one restricts from the beginning to Eq. (41) with $B_{i}=0$.

The relative difference between the tricritical values for $B_{\star}=1 \mathrm{GeV}$ and $B_{\star} \rightarrow \infty$ is found to be about a few percent. This indicates a controlled renormalization scale dependence and allows us from now on to work in a simplified picture in the $B_{\star} \rightarrow \infty$ limit. In particular, in this limit, we can associate a potential to the Euclidean localization, such that $V^{\prime}(B)=\mathcal{R}_{r}(B, 0)$. We now employ this simpler setting to move away from the chiral limit. ${ }^{8}$

For a nonzero bare mass, chiral symmetry is explicitly broken. As already mentioned, the second-order transition line turns into a crossover and the tricritical point into a critical point. Unlike the CEP, the crossover line has no

\footnotetext{
${ }^{7}$ This equation does not become trivial in the limit $B_{\star} \rightarrow \infty$ because, in this case, the $B_{\star}$-dependence of $1 / g^{2}$ is canceled by the corresponding $B_{\star}$-dependence hidden in $\tilde{F}$. We also mention that the equation obtained in the $B_{\star} \rightarrow \infty$ limit is nothing but the one we would have obtained by applying the naïve renormalization and sending the cutoff to infinity. Indeed the remaining cutoff dependence in the equation for $B_{i}$, only present in the term containing $1 / g_{0}^{2}$, would enforce $B_{i} \rightarrow 0$ as $\Lambda \rightarrow \infty$.

${ }^{8}$ We mention that, were we not to consider the simplifying limit $B_{\star} \rightarrow \infty$, certain properties would remain $B_{\star}$-independent, such as any property along the $T=0$ or $\mu=0$ axes. This includes the crossover temperature at $\mu=0$ or the values for $\mu_{\mathrm{ls}}, \mu_{\mathrm{first}}$ and $\mu_{\mathrm{us}}$ for any $H$, as well as the function $m_{+}(H)$ discussed below.
} 

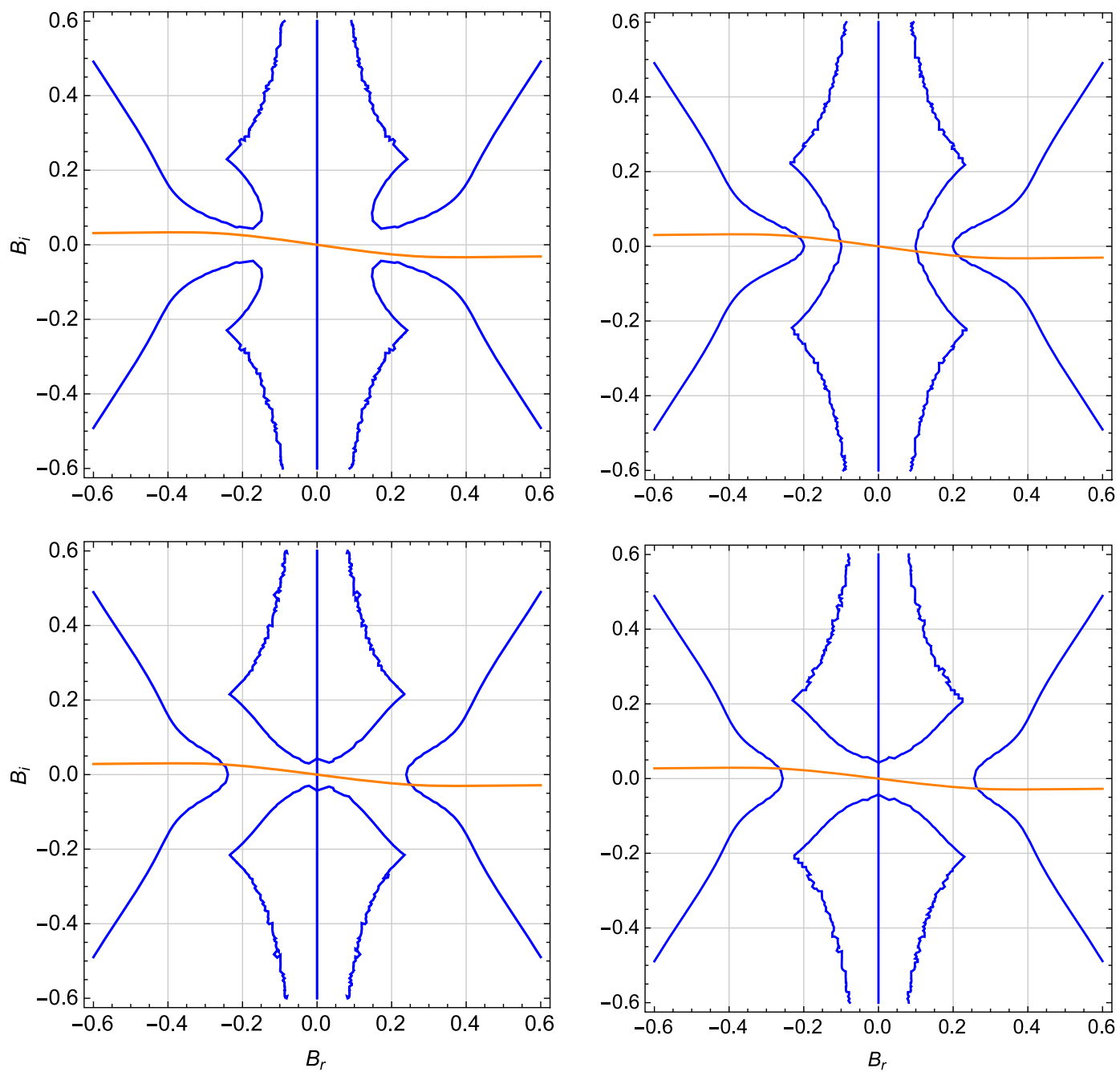

FIG. 5. 0-level plots of the gap equations in the chiral limit in the plane $\left(B_{r}, B_{i}\right)$ for decreasing temperatures, with $m=0.5, B_{0}=0.3$, and $B_{\star}=1$ (all units in $\mathrm{GeV}$ ). We have also chosen $\mu=0.33$ and $T$ takes the values $0.055,0.053,0.050$, and 0.048 . The wiggling in the curves is due to the presence of singularities of the gap equations in the plane $\left(B_{r}, B_{i}\right)$ that lead to additional 0 's of $\mathcal{R}_{r}$ or $\mathcal{R}_{i}$ (not visible). Fortunately, the actual solutions of the gap equations, corresponding to simultaneous 0 's of $\mathcal{R}_{r}$ and $\mathcal{R}_{i}$, are located far from these regions.

unique definition. Moreover, there exist as many crossover lines as there are order parameters. In what follows, we define the crossover temperature by the inflection of $B$ as a function of the temperature. With this choice, we can determine for which value of $H$ the crossover temperature of the quark mass function becomes $T_{\chi}=170 \mathrm{MeV}$ in the limit of vanishing chemical potential, which is the value found by lattice simulations at the physical point for two flavors [49]. Within our approach, we treat this particular value of $H$ to correspond to the physical point, $H_{\text {phys. }}$. We can determine it conveniently as follows. Denoting by $H=$ $\mathcal{R}(B)$ the gap equation in both localization schemes and taking two $T$-derivatives, we have

$$
0=\frac{\partial \mathcal{R}}{\partial B} \frac{d B}{d T}+\frac{\partial \mathcal{R}}{\partial T}
$$

$0=\frac{\partial^{2} \mathcal{R}}{\partial B^{2}}\left(\frac{d B}{d T}\right)^{2}+2 \frac{\partial^{2} \mathcal{R}}{\partial B \partial T} \frac{d B}{d T}+\frac{\partial^{2} \mathcal{R}}{\partial T^{2}}+\frac{\partial \mathcal{R}}{\partial B} \frac{d^{2} B}{d T^{2}}$

Imposing the inflection condition $d^{2} B / d T^{2}=0$ and upon plugging (50) into (51), we arrive at the following condition,

$0=\frac{\partial^{2} \mathcal{R}}{\partial B^{2}}\left(\frac{\partial \mathcal{R}}{\partial T}\right)^{2}-2 \frac{\partial^{2} \mathcal{R}}{\partial B \partial T} \frac{\partial \mathcal{R}}{\partial B} \frac{\partial \mathcal{R}}{\partial T}+\frac{\partial^{2} \mathcal{R}}{\partial T^{2}}\left(\frac{\partial \mathcal{R}}{\partial B}\right)^{2}$,

which we can solve for $B$, given the expected crossover temperature. Knowing the crossover value of $B$, we can then determine $H_{\text {phys }}$ from the gap equation. For the physical localization, we find $H_{\text {phys }}=33 \mathrm{MeV}$ whereas for the 
TABLE II. Coordinates of the critical point in the phase diagram at the physical point, $H_{\text {phys }}$. All values for $m=500$ and in $\mathrm{MeV}$. We compare our findings with various literature model computations of the QCD CEP.

\begin{tabular}{lcc}
\hline \hline Models for CEP & $\mu_{c}^{\text {phys }}$ & $T_{c}^{\text {phys }}$ \\
\hline Physical localization & 389 & 35 \\
Euclidean localization & 427 & 22 \\
Fischer et al. [50] & 168 & 115 \\
Fu et al. [25] & 210 & 117 \\
Hatta et al. [12] & 279 & 95 \\
Tripolt et al. [51] & 293 & 10 \\
Ayala et al. [52] & $315-349$ & $18-45$ \\
Cui et al. [53] & 245 & 38 \\
Yokota et al. [54] & 287 & 5 \\
Contrera et al. [55] & 319 & 70 \\
Knaute et al. [56] & 204 & 112 \\
Antoniou et al. [57] & 256 & 150 \\
Scavenius et al. [58] L $\sigma \mathrm{M}$ & 207 & 99 \\
Scavenius et al. [58] NJL & 332 & 46 \\
Costa et al. [48] & 332 & 80 \\
Kovacs et al. [59] & 320 & 63 \\
\hline \hline
\end{tabular}

Euclidean localization, we find $H_{\text {phys }}=10 \mathrm{MeV}$. Once $H_{\text {phys }}$ is determined, we can then locate the critical point in the associated phase diagram; see Table II.

As can be seen, the community has not yet reached a ballpark consensus on the location of the CEP in the QCD phase diagram and a wide range of results seem permissible at this point. Our numbers do certainly fall within the group of lower temperatures and larger chemical potentials.

Finally, one can study how our findings for the phase diagram depend on the gluon mass of the CF model. While $m=500 \mathrm{MeV}$ is the value that globally works best in both the pure Yang-Mills as well as the unquenched sector, it is nonetheless insightful to vary it as a free parameter. Thereby, for each value of $m$, we always insist on fixing the coupling such that we keep the $T=\mu=0$ solution $B_{0}$ fixed at $300 \mathrm{MeV}$, in the chiral limit. Away from the chiral limit, we only vary $H$, without further changing $g$.

In Fig. 6, we display the position of the tricritical point in the chiral limit as the CF mass parameter is varied. As can be seen, the obtained trajectories are qualitatively quite different depending on the considered localization scheme, although for $m=500 \mathrm{MeV}$, the tricritical points are not so far apart, in particular, in temperature values. Interestingly, while in all localization schemes considered, the gluon mass can never exceed an upper limit $m_{+}$, in the Euclidean localization, one might take $m \rightarrow 0$ without losing the tricritical point, so $m_{-}=0$ in this case.

As before the definition of $m_{ \pm}$is trivially extended to the case of nonzero $H$, where the tricritical point is replaced by a CEP. We then show our results for these values in dependence of the symmetry-breaking parameter $H$ in Fig. 7 and in comparison with the findings in the physical localization. Here, we iterate our observation that the

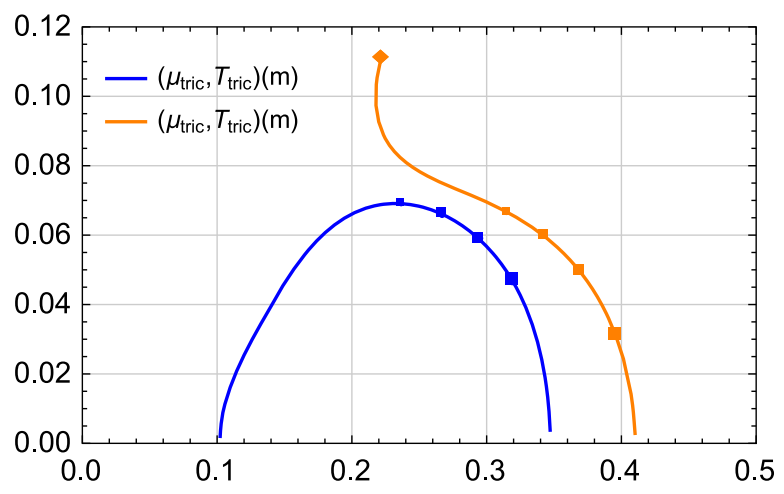

FIG. 6. Position of the tricritical points upon varying the gluon mass in the two considered localizations (physical in blue, Euclidean in orange). The various points correspond to $m=$ $x \times 500 \mathrm{MeV}$, with $x=1,1.25,1.5$ and 1.75 . The diamond point on the Euclidean localization curve corresponds to $m=0$ and its coordinates are $\mu=222 \mathrm{MeV}$ and $T=112 \mathrm{MeV}$. At the other end of the curve, we have $\mu=410 \mathrm{MeV}$ attained for $m=937 \mathrm{MeV}$.

existence of a CEP puts an upper bound on the allowed for values for the gluon mass in both localization schemes considered, whereas a lower bound only exists for the physical one.

\section{Chiral condensate}

As mentioned previously, the mostly used order parameter for the chiral transition is not the constituent quark mass but the chiral condensate. Within the localized schemes considered here, it is natural to approximate the chiral condensate as

$$
\sigma=-4 N_{c} N_{f} B J_{B}
$$

with

$$
J_{B} \equiv \int_{\hat{Q}}^{T} \frac{1}{Q_{i \mu}^{2}+B^{2}}
$$

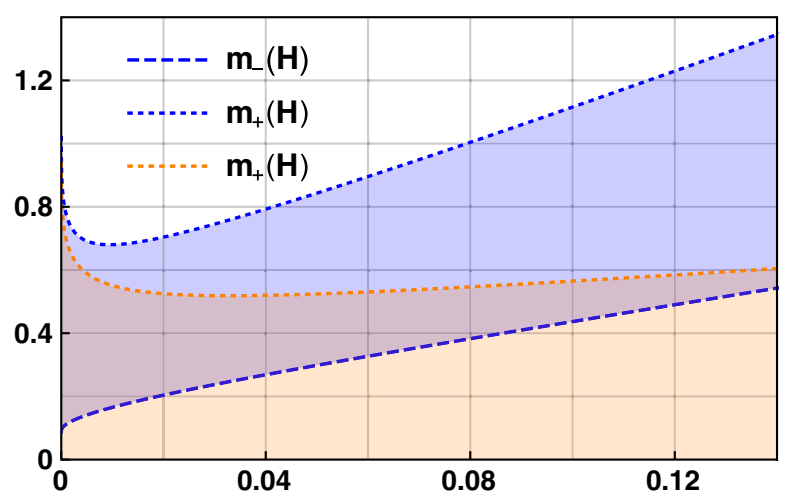

FIG. 7. Comparison of the functions $m_{ \pm}(H)$ in the physical and Euclidean localizations. The respective shaded areas denote the parameter values compatible with the existence of a CEP. 

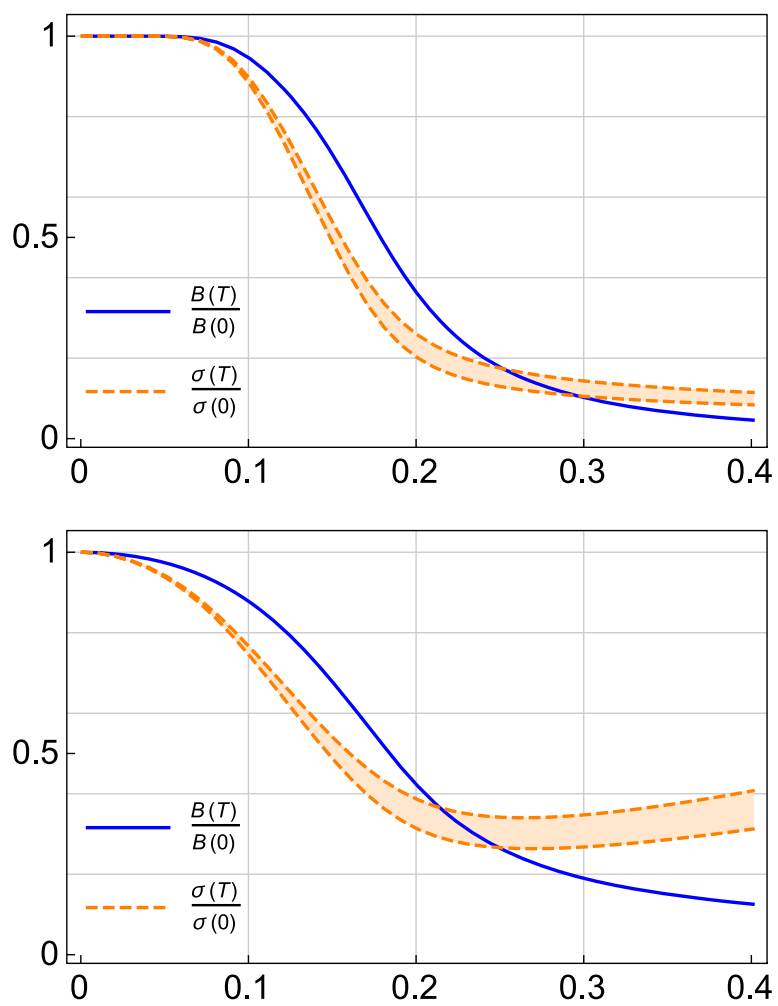

FIG. 8. Mass and renormalized chiral condensate (top plot, physical localization; bottom plot, Euclidean localization) as functions of the temperature and at $\mu=0$. The band for the renormalized chiral condensate is obtained by varying the renormalization scale $\bar{\mu}$ in $\bar{J}_{B}$ by $\pm 20 \%$ around $1 \mathrm{GeV}$. Both plots are obtained by adjusting $H$ such that the crossover temperature associated to $B$ is $170 \mathrm{MeV}$.

At this level of description, we can adopt an ad hoc renormalization of the condensate by removing the divergence in $J_{B}$, up to the scale in the logarithm of the vacuum contribution of $J_{B}$ which makes the renormalized condensate $\bar{\sigma}$ a scale-dependent quantity,

$$
\bar{\sigma}=-4 N_{c} N_{f} B \bar{J}_{B},
$$

with

$\bar{J}_{B} \equiv-\frac{B^{2}}{16 \pi^{2}}\left[\ln \frac{\bar{\mu}^{2}}{B^{2}}+1\right]+\frac{1}{4 \pi^{2}} \int_{0}^{\infty} d q \frac{q^{2}}{\varepsilon_{q}^{B}}\left[n_{\varepsilon_{q}^{B}-\mu}^{(+)}+n_{\varepsilon_{q}^{B}+\mu}^{(+)}\right]$.

The condensate (55) is plotted, together with the quark mass, as functions of the temperature in Fig. 8. To get some intuition on the behavior of $\bar{\sigma}$, consider the physically localized gap equation that we rewrite identically as

$$
H=4 \pi^{2} B\left[\frac{\bar{J}_{m}-\bar{J}_{B}}{m^{2}-B^{2}}-\frac{\bar{J}_{m}^{\mathrm{vac}}-\bar{J}_{B_{0}}^{\mathrm{vac}}}{m^{2}-B_{0}^{2}}\right] .
$$

In the limit $T \rightarrow \infty$, the tadpole sum-integral $\bar{J}_{m}$ grows like $\sim T^{2} / 12$, which is only counterbalanced provided $B$ vanishes as $1 / T^{2}$, in which case we also have $\bar{J}_{B} \sim$ $-T^{2} / 24$. Putting all the pieces together, we find

$$
B \sim \frac{2}{\pi^{2}} \frac{m^{2} H}{T^{2}}
$$

and thus

$$
\bar{\sigma} \rightarrow \frac{N_{c} N_{f}}{3 \pi^{2}} m^{2} H
$$

In the Euclidean localization the condensate is found to grow with $T^{2}$ at large $T$, which is clearly an artefact. ${ }^{9}$ Despite this feature, in both cases, we can define a crossover temperature associated to the inflexion point of the chiral condensate as a function of the temperature. It is found to be lower than the corresponding crossover temperature for $B$. We can also try to fine-tune the value of $H$ to bring the crossover temperature for the condensate to the lattice value of $170 \mathrm{MeV}$. To this purpose, consider the equation $d^{2} \bar{\sigma} / d T^{2}=0$. Seeing $\bar{\sigma}$ as a function of $B$ and $T$, this equation rewrites

$$
\begin{aligned}
0= & \left(\frac{\partial \mathcal{R}}{\partial T}\right)^{2}\left(\frac{\partial^{2} \mathcal{R}}{\partial B^{2}} \frac{\partial \sigma}{\partial B}-\frac{\partial^{2} \sigma}{\partial B^{2}} \frac{\partial \mathcal{R}}{\partial B}\right) \\
& -2 \frac{\partial \mathcal{R}}{\partial T} \frac{\partial \mathcal{R}}{\partial B}\left(\frac{\partial^{2} \mathcal{R}}{\partial B \partial T} \frac{\partial \sigma}{\partial B}-\frac{\partial^{2} \sigma}{\partial B \partial T} \frac{\partial \mathcal{R}}{\partial B}\right) \\
& +\left(\frac{\partial \mathcal{R}}{\partial B}\right)^{2}\left(\frac{\partial^{2} \mathcal{R}}{\partial T^{2}} \frac{\partial \sigma}{\partial B}-\frac{\partial^{2} \sigma}{\partial T^{2}} \frac{\partial \mathcal{R}}{\partial B}\right),
\end{aligned}
$$

which we use again to determine the crossover value for $B$, assuming a crossover temperature $T_{\chi}=170 \mathrm{MeV}$. The corresponding value of $H$ is then obtained from the gap equation. In the physical localization, we find $H_{\text {phys }}=$ $117 \mathrm{MeV}$, and a CEP located at (504 MeV, $11 \mathrm{MeV})$, but the value of $B$ gets suspiciously close to the bound $B=m$. In the Euclidean localization, it seems not to be possible to reach these transition temperatures, at least at this level of approximation.

\section{CONCLUSION}

We have investigated the phase diagram of QCD with light quarks in the context of an effective approach proposed in Ref. [40], based on the CF model, using a double expansion in the pure gauge coupling and in $1 / N_{c}$. At leading order, this amounts to solving a rainbow equation for the quark propagator with definite (tree-level) expression for the one-gluon exchange. Similar rainbow

\footnotetext{
${ }^{9}$ The coefficient is proportional to $H$ however in such a way that the condensate vanishes in the chiral limit, in the hightemperature region.
} 
equations have been considered in the past. The key point of the present work is to systematically justify the employed approximation on the basis of identified small parameters in QCD.

To allow for a semianalytic grasp of the corresponding equations and since our first aim is a qualitative survey of what to expect when the equations are solved in full glory, we have used some simplifying approximations, in particular, the localization scheme discussed in [41,42], which we have extended to the present context. For the parameters used here, the leading-order results agree well with those of effective quark-meson models when the chiral anomaly is neglected [26,30]. Although subleading in $1 / N_{c}$, the latter and meson fluctuations are important to correctly determine the phase structure in the Columbia plot $[7,30,60]$. In principle, they can be systematically included at next-toleading order in the present expansion scheme.

Although we used here simplified versions of the complete rainbow equation (1), we expect, inspired by the vacuum case, that our main results are robust against a more complete treatment of the original equation. In particular, we have tested that our results do not depend much on the type of localization we use. One notable exception is the fate of the (tri)critical point as the gluon mass is taken to 0 . In some scenarios, the existence of a CEP seems to require a nonzero gluon mass. It is interesting to investigate this point further by solving the original set of equations (10)-(13), which can be done with existing technology; see, e.g., Refs. [13,14].

Yet another interesting extension of the present work is to include the order parameter of the deconfinement transition, the Polyakov loop, in the spirit of Refs. [36-38], which would allow one to study the interplay between the chiral and deconfinement phase transition across the Columbia plot $[27,61,62]$.

\section{ACKNOWLEDGMENTS}

We thank Zs. Szép for interesting discussions and helpful remarks concerning the manuscript.

\section{APPENDIX A: SYMMETRIES OF THE QUARK PROPAGATOR}

The full propagator $S$ is a function of the external momentum variables $\omega$ and $\vec{p}$ as well as $\mu$ and $T$. Dropping the explicit $T$-dependence for notational simplicity, and assuming isotropy, its tensor structure can decomposed as

$$
\begin{aligned}
S(\omega, \vec{p} ; \mu)= & S_{1} \mathbb{1}+S_{2} \gamma_{5}+S_{3} \gamma_{0}+S_{4} \gamma_{0} \gamma_{5} \\
& +S_{5} \hat{p} \cdot \vec{\gamma}+S_{6} \hat{p} \cdot \vec{\gamma} \gamma_{5}+S_{7} \gamma_{0} \hat{p} \cdot \vec{\gamma},
\end{aligned}
$$

where $S_{i}=S_{i}(\omega, p ; \mu), p \equiv|\vec{p}|$, and $\hat{p} \equiv \vec{p} / p$. Under a parity transformation

$$
\begin{aligned}
& S(\omega, \vec{p} ; \mu) \rightarrow \gamma_{0} S(\omega,-\vec{p} ; \mu) \gamma_{0} \\
& =S_{1} \mathbb{1}-S_{2} \gamma_{5}+S_{3} \gamma_{0}-S_{4} \gamma_{0} \gamma_{5} \\
& \quad+S_{5} \hat{p} \cdot \vec{\gamma}-S_{6} \hat{p} \cdot \vec{\gamma} \gamma_{5}+S_{7} \gamma_{0} \hat{p} \cdot \vec{\gamma},
\end{aligned}
$$

and thus parity invariance implies $S_{2}=S_{4}=S_{6}=0$. In fact, in what follows, it is convenient to use the parametrization

$S(\omega, \vec{p} ; \mu)=\tilde{B} \mathbb{1}+(i \omega-\mu) \gamma_{0} \tilde{A}_{0}+i \vec{p} \cdot \vec{\gamma} \tilde{A}_{v}+i \gamma_{0} \vec{p} \cdot \vec{\gamma} \tilde{C}$.

Under charge conjugation

$$
\begin{aligned}
& S(\omega, \vec{p} ; \mu) \rightarrow \gamma_{2} \gamma_{0} S(-\omega,-\vec{p} ;-\mu)^{\mathrm{t}} \gamma_{0} \gamma_{2} \\
& =\tilde{B}(-\omega, p ;-\mu) \mathbb{1}+(i \omega-\mu) \gamma_{0} \tilde{A}_{0}(-\omega, p ;-\mu) \\
& \quad+i \vec{p} \cdot \vec{\gamma} \tilde{A}_{v}(-\omega, p ;-\mu)+i \gamma_{0} \vec{p} \cdot \vec{\gamma} \tilde{C}(-\omega, p ;-\mu),
\end{aligned}
$$

where we have used $\gamma_{2} \gamma_{0} \gamma_{\mu}^{\mathrm{t}} \gamma_{0} \gamma_{2}=-\gamma_{\mu}$, valid in the particular (Weyl) representation of the $\gamma_{\mu}$ matrices considered here. Charge conjugation invariance then implies

$$
\tilde{X}(-\omega, p ;-\mu)=\tilde{X}(\omega, p ; \mu)
$$

for any of the components $\tilde{X}=\tilde{A}_{0}, \tilde{A}_{v}, \tilde{B}, \tilde{C}$. Similarly, under complex conjugation

$$
\begin{aligned}
& S(\omega, \vec{p} ; \mu) \rightarrow \gamma_{3} \gamma_{1} S\left(-\omega,-\vec{p} ; \mu^{*}\right)^{*} \gamma_{1} \gamma_{3} \\
& =\tilde{B}\left(-\omega, p ; \mu^{*}\right)^{*} \mathbb{1}+(i \omega-\mu) \gamma_{0} \tilde{A}_{0}\left(-\omega, p ; \mu^{*}\right)^{*} \\
& \quad+i \vec{p} \cdot \vec{\gamma} \tilde{A}_{v}\left(-\omega, p ; \mu^{*}\right)^{*}+i \gamma_{0} \vec{p} \cdot \vec{\gamma} \tilde{C}\left(-\omega, p ; \mu^{*}\right)^{*},
\end{aligned}
$$

where we have used $\gamma_{3} \gamma_{1} \gamma_{\mu}^{*} \gamma_{1} \gamma_{3}=\gamma_{\mu}$, valid, again, in the Weyl representation. It follows that

$$
\tilde{X}\left(-\omega, p ; \mu^{*}\right)^{*}=\tilde{X}(\omega, p ; \mu)
$$

for any of the components $\tilde{X}=\tilde{A}_{0}, \tilde{A}_{v}, \tilde{B}, \tilde{C}$. Combining (A5) and (A7), we also obtain

$$
\tilde{X}\left(\omega, p ;-\mu^{*}\right)^{*}=\tilde{X}(\omega, p ; \mu) .
$$

In particular, all components are real in the case of an imaginary chemical potential. In the case of a real chemical potential, these components become complex, the real and imaginary parts, corresponding to the frequency even and odd parts, $(\tilde{X}(\omega, p ; \mu)+\tilde{X}(-\omega, p ; \mu)) / 2$ and $(\tilde{X}(\omega, p ; \mu)-\tilde{X}(-\omega, p ; \mu)) / 2 i$, respectively. 


\section{APPENDIX B: RETARDED GREEN'S FUNCTION AT FINITE $\mu$}

We briefly recall the origin of Eq. (27), considering, for simplicity, the case of a charged scalar field. The physical retarded propagator is defined as

$$
G_{\mathrm{ret}}^{\mathrm{phys}}(t) \equiv-i \frac{\Theta(t)}{Z} \operatorname{tr} e^{-\beta(H+\mu Q)}\left[\varphi_{H}(t), \varphi^{\dagger}(0)\right],
$$

where $Z \equiv \operatorname{tr} e^{-\beta(H+\mu Q)}$ denotes the grand-canonical partition function, and $\varphi_{H}(t)=e^{i H t} \varphi(0) e^{-i H t}$ is the Heisenberg field, evolving according to $H$ and not $H+\mu Q$. Let us note that we use an unconventional sign for the chemical potential (to be consistent with our choice in $[37,38]$ ) while keeping the usual convention for the charge, such that $[Q, \varphi]=-\varphi$ and $\left[Q, \varphi^{\dagger}\right]=\varphi^{\dagger}$.

Now, we relate the physical retarded propagator to the Matsubara propagator defined as $(0<\tau<\beta)$

$$
G_{\mathrm{Mat}}(\tau) \equiv \frac{1}{Z} \operatorname{tr} e^{-\beta(H+\mu Q)} \varphi_{H+\mu Q}(-i \tau) \varphi^{\dagger}(0),
$$

where now the Heisenberg field evolves in imaginary time, according to $H+\mu Q$, not $H$. The reason for defining the Matsubara propagator in this way is that it possesses a simple functional integral representation.
The relation between the two propagators $G_{\mathrm{ret}}^{\text {phys }}(t)$ and $G_{\text {Mat }}(\tau)$ is now most easily derived by introducing an auxiliary retarded propagator

$$
G_{\mathrm{ret}}(t) \equiv-i \frac{\Theta(t)}{Z} \operatorname{tr} e^{-\beta(H+\mu Q)}\left[\varphi_{H+\mu Q}(t), \varphi^{\dagger}(0)\right] .
$$

Inserting a complete basis of states under the trace, this relation in Fourier space is found to be

$$
G_{\text {ret }}(\omega)=G_{\text {Mat }}\left(\omega_{n} \rightarrow-i \omega+0^{+}\right) .
$$

Moreover, from the commutators of $Q$ with $\varphi$ or $\varphi^{\dagger}$, one obtains

$$
G_{\mathrm{ret}}^{\mathrm{phys}}(t)=e^{i \mu t} G_{\mathrm{ret}}(t)
$$

and thus

$$
G_{\mathrm{ret}}^{\text {phys }}(\omega)=G_{\mathrm{ret}}(\omega+\mu) .
$$

Combining (B4) and (B6), we arrive at the desired result,

$$
G_{\text {ret }}^{\text {phys }}(\omega)=G_{\text {Mat }}\left(\omega_{n} \rightarrow-i(\omega+\mu)+0^{+}\right) .
$$

[1] K. Fukushima and T. Hatsuda, Rep. Prog. Phys. 74, 014001 (2011).

[2] S. Weissenborn, I. Sagert, G. Pagliara, M. Hempel, and J. Schaffner-Bielich, Astrophys. J. 740, L14 (2011).

[3] S. Borsányi et al., Nature (London) 539, 69 (2016).

[4] Y. Aoki, Z. Fodor, S. D. Katz, and K. K. Szabo, Phys. Lett. B 643, 46 (2006).

[5] A. Bazavov et al., Phys. Rev. D 85, 054503 (2012).

[6] M. D'Elia, Nucl. Phys. A982, 99 (2019).

[7] R. D. Pisarski and F. Wilczek, Phys. Rev. D 29, 338 (1984).

[8] P. de Forcrand, Proc. Sci., LAT2009 (2009) 010 [arXiv: 1005.0539].

[9] M. A. Stephanov, Prog. Theor. Phys. Suppl. 153, 139 (2004); Int. J. Mod. Phys. A 20, 4387 (2005).

[10] B. Mohanty and J. Serreau, Phys. Rep. 414, 263 (2005).

[11] M. A. Stephanov, Phys. Rev. Lett. 102, 032301 (2009).

[12] Y. Hatta and T. Ikeda, Phys. Rev. D 67, 014028 (2003).

[13] C. D. Roberts and S. M. Schmidt, Prog. Part. Nucl. Phys. 45, S1 (2000).

[14] C. S. Fischer, Prog. Part. Nucl. Phys. 105, 1 (2019).

[15] K. Fukushima and C. Sasaki, Prog. Part. Nucl. Phys. 72, 99 (2013).

[16] H. T. Ding, F. Karsch, and S. Mukherjee, Int. J. Mod. Phys. E 24, 1530007 (2015).
[17] B. Mohanty (STAR Collaboration), J. Phys. G 38, 124023 (2011).

[18] P. Senger, Eur. Phys. J. A 52, 217 (2016).

[19] T. Ablyazimov et al. (CBM Collaboration), Eur. Phys. J. A 53, 60 (2017).

[20] T. Sakaguchi (J-PARC-HI Collaboration), Nucl. Phys. A967, 896 (2017).

[21] Z. Fodor et al., arXiv:1807.09862.

[22] C. S. Fischer and J. Luecker, Phys. Lett. B 718, 1036 (2013).

[23] C. S. Fischer, L. Fister, J. Luecker, and J. M. Pawlowski, Phys. Lett. B 732, 273 (2014).

[24] G. Eichmann, C. S. Fischer, and C. A. Welzbacher, Phys. Rev. D 93, 034013 (2016).

[25] W.j. Fu, J. M. Pawlowski, and F. Rennecke, arXiv:1909 .02991 .

[26] A. Jakovác, A. Patkós, Z. Szép, and P. Szépfalusy, Phys. Lett. B 582, 179 (2004); Acta Phys. Hung. A 22, 355 (2005).

[27] B. J. Schaefer, J. M. Pawlowski, and J. Wambach, Phys. Rev. D 76, 074023 (2007).

[28] K. Fukushima, Phys. Rev. D 77, 114028 (2008); 78, 039902 (E) (2008).

[29] T. K. Herbst, J. M. Pawlowski, and B. J. Schaefer, Phys. Lett. B 696, 58 (2011); Phys. Rev. D 88, 014007 (2013). 
[30] S. Resch, F. Rennecke, and B. J. Schaefer, Phys. Rev. D 99, 076005 (2019).

[31] G. Curci and R. Ferrari, Nuovo Cimento A 32, 151 (1976).

[32] M. Tissier and N. Wschebor, Phys. Rev. D 82, 101701 (2010); 84, 045018 (2011).

[33] I. L. Bogolubsky, E. M. Ilgenfritz, M. Muller-Preussker, and A. Sternbeck, Phys. Lett. B 676, 69 (2009).

[34] J. Serreau and M. Tissier, Phys. Lett. B 712, 97 (2012).

[35] U. Reinosa, J. Serreau, M. Tissier, and N. Wschebor, Phys. Rev. D 96, 014005 (2017).

[36] U. Reinosa, J. Serreau, M. Tissier, and N. Wschebor, Phys. Lett. B 742, 61 (2015); Phys. Rev. D 91, 045035 (2015); 93, 105002 (2016).

[37] U. Reinosa, J. Serreau, and M. Tissier, Phys. Rev. D 92, 025021 (2015).

[38] J. Maelger, U. Reinosa, and J. Serreau, Phys. Rev. D 97, 074027 (2018); 98, 094020 (2018).

[39] J. I. Skullerud, P. O. Bowman, A. Kizilersu, D. B. Leinweber, and A. G. Williams, J. High Energy Phys. 04 (2003) 047.

[40] M. Peláez, U. Reinosa, J. Serreau, M. Tissier, and N. Wschebor, Phys. Rev. D 96, 114011 (2017).

[41] U. Reinosa and Zs. Szép, Phys. Rev. D 85, 045034 (2012).

[42] G. Markó, U. Reinosa, and Zs. Szép, Phys. Rev. D 92 , 125035 (2015).

[43] M. Laine and A. Vuorinen, Lect. Notes Phys. 925, 1 (2016).

[44] T. D. Cohen, Phys. Rev. Lett. 91, 222001 (2003).

[45] G. Markó, U. Reinosa, and Zs. Szép, Phys. Rev. D 90, 125021 (2014).
[46] S. X. Qin, L. Chang, H. Chen, Y. x. Liu, and C. D. Roberts, Phys. Rev. Lett. 106, 172301 (2011).

[47] B. J. Schaefer and J. Wambach, Nucl. Phys. A757, 479 (2005).

[48] P. Costa, M. C. Ruivo, and C. A. de Sousa, Phys. Rev. D 77, 096001 (2008).

[49] F. Karsch, E. Laermann, and A. Peikert, Nucl. Phys. B605, 579 (2001).

[50] C. S. Fischer, J. Luecker, and C. A. Welzbacher, Phys. Rev. D 90, 034022 (2014).

[51] R. A. Tripolt, L. von Smekal, and J. Wambach, Phys. Rev. D 90, 074031 (2014).

[52] A. Ayala, S. Hernandez-Ortiz, and L. A. Hernandez, Rev. Mex. Fis. 64, 302 (2018).

[53] Z. F. Cui, J. L. Zhang, and H. S. Zong, Sci. Rep. 7, 45937 (2017).

[54] T. Yokota, T. Kunihiro, and K. Morita, arXiv:1611.06669.

[55] G. A. Contrera, A. G. Grunfeld, and D. Blaschke, Eur. Phys. J. A 52, 231 (2016).

[56] J. Knaute, R. Yaresko, and B. Kämpfer, Phys. Lett. B 778, 419 (2018).

[57] N. G. Antoniou, F. K. Diakonos, X. N. Maintas, and C. E. Tsagkarakis, Phys. Rev. D 97, 034015 (2018).

[58] O. Scavenius, A. Mocsy, I. N. Mishustin, and D. H. Rischke, Phys. Rev. C 64, 045202 (2001).

[59] P. Kovacs and Z. Szep, Phys. Rev. D 77, 065016 (2008).

[60] J. T. Lenaghan, Phys. Rev. D 63, 037901 (2001).

[61] K. Fukushima, Phys. Lett. B 591, 277 (2004).

[62] A. Folkestad and J. O. Andersen, Phys. Rev. D 99, 054006 (2019). 